\title{
Uncertainty estimates for magnetic relaxation times and magnetic relaxation parameters
}

Daniel Reta ${ }^{\mathrm{a},{ }^{*}}$ and Nicholas F. Chilton ${ }^{\mathrm{a}, *}$

${ }^{a}$ School of Chemistry, The University of Manchester, Oxford Road, M13 9PL, UK

\section{Abstract}

The use of alternating current $(A C)$ magnetometry to measure magnetic relaxation times is one of the most fundamental measurements for characterising single-molecule magnets (SMMs). These measurements, performed as a function of frequency, temperature and magnetic field, give vital information on the underlying magnetic relaxation process(es) occurring in the material. The magnetic relaxation times are usually fitted to model functions derived from spin-phonon coupling theories that allow characterisation of the mechanisms of magnetic relaxation. The parameters of these relaxation models are then often compared between different molecules in order to find trends with molecular structure that may guide the field to the next breakthrough. However, such meta-analyses of the model parameters are doomed to over-interpretation unless uncertainties in the model parameters can be quantified. Here we determine a method for obtaining uncertainty estimates in magnetic relaxation times from AC experiments, and provide a program called CC-FIT2 for fitting experimental $A C$ data as well as the resulting relaxation times, to obtain relaxation parameters with accurate uncertainties. Applying our approach to three archetypal families of high-performance dysprosium(III) SMMs shows that accounting for uncertainties has a significant impact on the uncertainties of relaxation parameters, and that larger uncertainties appear to correlate with crystallographic disorder in the compounds studied. We suggest that this type of analysis should become routine in the community.

\section{Introduction}

Single-molecule magnets (SMMs) are superparamagnetic molecules with large energy barriers to the inversion of their magnetic moment, $U_{\text {eff, }}$ such that their magnetic relaxation is slow enough to observe memory effects such as magnetic hysteresis. ${ }^{1}$ At high temperatures, molecules change spin state and overcome the $U_{\text {eff }}$ barrier by exchanging energy with the phonon bath through first-order spin-phonon coupling (known as the Orbach process), ${ }^{2}$ but as the temperature is decreased this process becomes exponentially slower. At low temperatures, thermally-activated magnetic relaxation is more efficient via a second-order spin-phonon coupling process where pairs of phonons exchange their energy difference with molecules (known as the Raman process); ${ }^{3,4}$ at even lower temperatures a temperature-independent quantum tunnelling of the magnetisation (QTM) is often observed. ${ }^{5}$

In the last few years, the $U_{\text {eff }}$ barrier has become so large for dysprosium(III)-based SMMs ${ }^{6-9}$ that the over-barrier Orbach relaxation process does not dominate in the temperature range where hysteresis can be observed, ${ }^{4}$ and memory effects have more to do with the Raman relaxation process. ${ }^{10}$ Despite the characteristic power-law temperature dependence of the Raman process being known for several decades, ${ }^{2}$ high-performance SMMs display a range of Raman parameters that do not appear to conform with predictions from traditional theories. ${ }^{10}$ Furthermore, while the physical origin of $U_{\text {eff }}$ is well accepted, ${ }^{4,11}$ there is as-yet no intuitive link between chemical structure and Raman relaxation and thus avenues for significant improvement cannot be predicted. To understand the physical origins of the Raman process, the behaviour of a wide range of different SMMs must be compared, ideally in families where structural parameters are changed systematically, but this can only be done when 
reliable uncertainties for the Raman parameters are known, to minimise possible correlations in the parameters and to exclude unreliable datasets. However, the largest uncertainties in model parameters rarely lie in the non-linear least-squares fitting of relaxation models, but rather in the underlying data - the magnetic relaxation times - which are frequently extracted from experiment using an empirical distribution model without comment on the associated uncertainties.

Herein we examine the distribution of relaxation times implicit in the generalised Debye model, and determine expressions for $1 \sigma$ and $2 \sigma$ uncertainties in the extracted relaxation times (see Results). We then present a new program for fitting the data from AC susceptibility experiments, that not only obtains uncertainties in the relaxation times, but also allows an interactive fit of the extracted data to obtain model parameters with reliable uncertainties (see Implementation). Finally, we apply our analysis to three archetypal families of high-performance dysprosium(III) SMMs to show that accounting for uncertainties in relaxation times has significant implications for the uncertainties of relaxation parameters, and that larger uncertainties appear to correlate with crystallographic disorder (see Discussion).

\section{Results}

Upon application of an AC magnetic field, the magnetic moment of a sample will oscillate in-phase with the field if its magnetic relaxation time, $\tau$, is short. If its relaxation time is long compared to the timescale of the oscillating field, then the sample cannot keep up with the field and the out-of-phase magnetic susceptibility becomes non-zero. The general expression for the AC magnetic susceptibility is a complex-valued quantity, where the in-phase component $\chi^{\prime}$ is the real part of Equation 1 , and the out-of-phase component $\chi^{\prime \prime}$ is the imaginary part; this is known as the Debye model. ${ }^{1}$

$$
\chi_{A C}(\omega)=\chi_{S}+\frac{\chi_{T}-\chi_{S}}{1+i \omega \tau}
$$

Here, $\chi_{T}$ is the isothermal susceptibility, $\chi_{S}$ is the adiabatic susceptibility, and $\omega$ is the angular frequency of the AC field (note $\omega=2 \pi v$, where $v$ is the linear frequency). In the low-frequency limit where $\omega \ll \tau^{-1}$, the sample can maintain equilibrium in the AC field and $\chi_{T}$ is measured, but in the high-frequency limit $\omega \gg \tau^{-1}$ then the magnetisation of the sample is fixed on the time-scale of $\omega$ and $\chi_{S}$ is measured (Figure 1 ). When the frequency of the $A C$ field is equal to the magnetic relaxation rate (i.e. $\omega=\tau^{-1}$ ) then the out-of-phase susceptibility peaks at a value of $\chi^{\prime \prime}=\left|\frac{1}{2}\left(\chi_{S}-\chi_{T}\right)\right|$, and the in-phase susceptibility changes from $\chi_{T}$ to $\chi_{S}$ (Figure 1 ). Thus, AC studies can measure magnetic relaxation times at a given temperature by varying the $A C$ frequency.

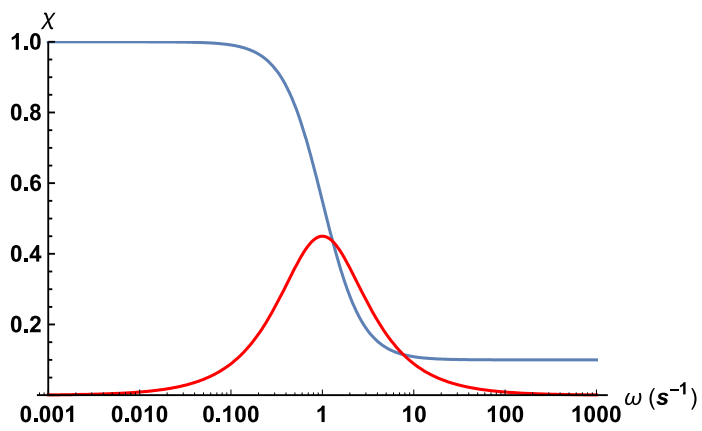


Figure 1 - In-phase AC susceptibility $\chi^{\prime}$ (blue) and out-of-phase AC susceptibility $\chi^{\prime \prime}$ (red) as a function of $\omega \cdot \chi_{S}=0.1, \chi_{T}=1$ and $\tau=1 \mathrm{~s}$.

For most SMMs however, the peak value of the out-of-phase component does not reach $\chi^{\prime \prime}=$ $\frac{1}{2}\left|\chi_{S}-\chi_{T}\right|$. This effect, usually ascribed to a distribution of relaxation times, can be emulated by using the generalised Debye model (Equation 2), ${ }^{1,12}$ where the parameter $\alpha$ empirically models the distribution. When $\alpha=0$ there is no distribution of relaxation times (i.e. the distribution in $\tau$ is a delta function and the original Debye model is recovered) and when $\alpha=1$ there is an infinitely flat distribution of relaxation times and $\chi^{\prime \prime}=0$. Thus, there is a monotonous but non-linear decrease in the peak value of $\chi^{\prime \prime}$ as a function of $\alpha$ (Equation 3 and Figure 2).

$$
\begin{array}{r}
\chi_{A C}(\omega)=\chi_{S}+\frac{\chi_{T}-\chi_{S}}{1+(i \omega \tau)^{1-\alpha}} \\
\chi_{\text {Peak }}^{\prime \prime}(\alpha)=\left(\chi_{T}-\chi_{S}\right) \frac{\cos \left[\frac{\pi \alpha}{2}\right]}{2\left(1+\sin \left[\frac{\pi \alpha}{2}\right]\right)}
\end{array}
$$
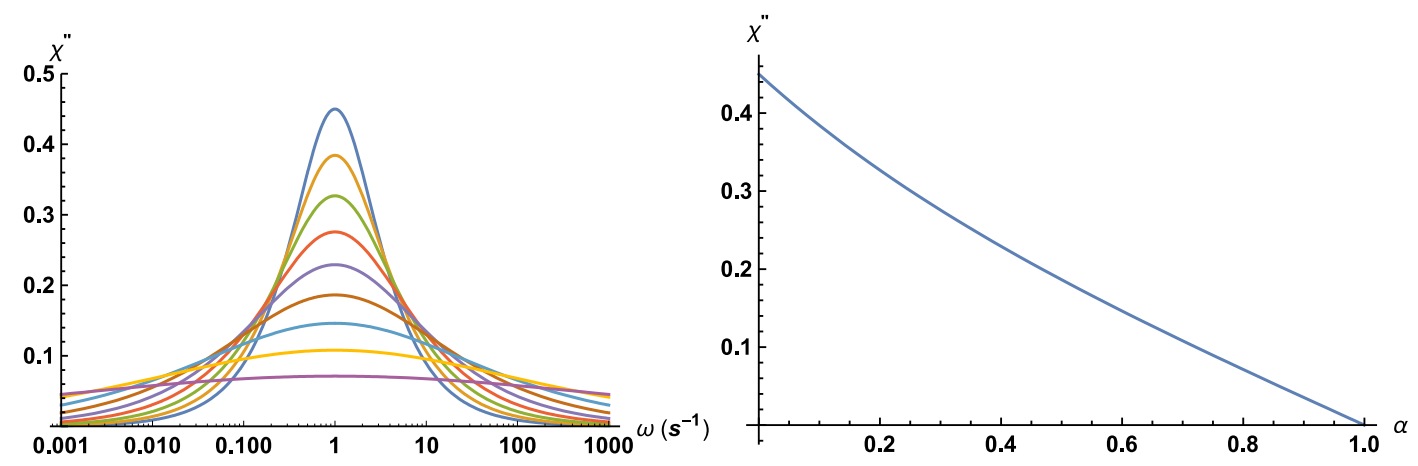

Figure 2 - (Left) Out-of-phase AC susceptibility $\chi^{\prime \prime}$ as a function of $\omega$, for different values of $\alpha$ from $\alpha=0$ (blue) to $\alpha=0.8$ (purple) in steps of 0.1 . (Right) Peak out-of-phase AC susceptibility $\chi^{\prime \prime}$ at $\omega=$ $\tau^{-1}$ as a function of $\alpha \cdot \chi_{S}=0.1, \chi_{T}=1$ and $\tau=1$.

Fuoss and Kirkwood ${ }^{13}$ have derived the distribution of relaxation times implied by the generalised Debye model (Equation 4), where $s=\ln \left[\frac{\tau}{\tau_{\mu}}\right]$ with $\tau_{\mu}$ being the mean relaxation time. To determine uncertainties in $\tau$ for given values of $\alpha$, we seek the symmetric integration region $-A \leq s \leq A$ that contains a fraction of the distribution equal to that within one or two standard deviations either side of the mean in the normal distribution (Equation 5, where $n$ is either 1 or 2 for $1 \sigma$ and $2 \sigma$, respectively). There is no analytical solution to this problem, so we determine numerical solutions for a range of $\alpha$ values (Figures 3 and S1); the form of these data are very well approximated by Equation 6 (note that this is simply an interpolating function with the correct limiting behaviour and not a model directly derived from the distribution). The associated uncertainty ranges in $\tau$ are given in Table 1 (note that the range for $2 \sigma$ is not simply double the range of $1 \sigma$ ).

$$
\rho_{\text {Fuoss-Kirkwood }}(s, \alpha)=\frac{\sin [\alpha \pi]}{2 \pi(\cosh [(1-\alpha) s]-\cos [\alpha \pi])}
$$




$$
\int_{-A}^{A} \rho_{\text {Fuoss-Kirkwood }}(s, \alpha) d s=\frac{1}{\sqrt{\pi}} \int_{-\frac{n}{\sqrt{2}}}^{\frac{n}{\sqrt{2}}} e^{-x^{2}} d x
$$

$$
A_{1 \sigma} \approx \frac{2.57 \tan \left[\frac{\alpha \pi}{2}\right]}{\alpha^{0.096}}
$$

$$
A_{2 \sigma} \approx \frac{5.52 \tan \left[\frac{\alpha \pi}{2}\right]}{\alpha^{0.30}}
$$
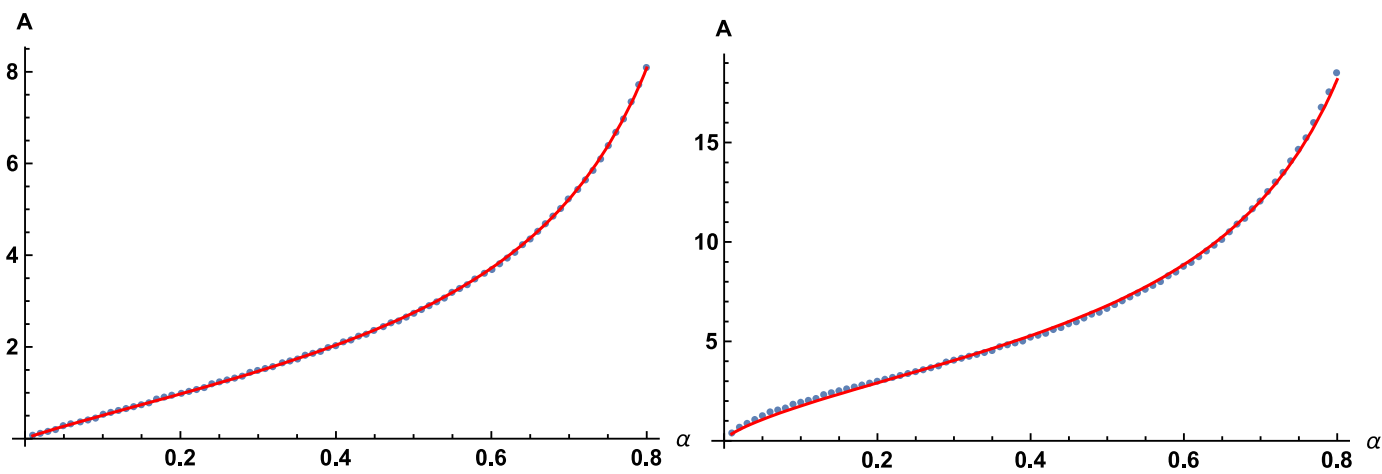

Figure 3 - Range of $\pm A$ required to encapsulate $1 \sigma$ (left) and $2 \sigma$ (right) populations as a function of $\alpha$ from the Fuoss-Kirkwood distribution. Red lines are best fits with $A_{1 \sigma} \approx \frac{2.57 \tan \left[\frac{\alpha \pi}{2}\right]}{\alpha^{0.096}}$ (left), $A_{2 \sigma} \approx$ $\frac{5.52 \tan \left[\frac{\alpha \pi}{2}\right]}{\alpha^{0.30}}$ (right).

Table 1 - Uncertainty ranges for $\tau$ based on different models

\begin{tabular}{|c|c|c|}
\hline Model & $\mathbf{1 \sigma}$ & $\mathbf{2 \sigma}$ \\
\hline Fuoss-Kirkwood & $\tau_{ \pm}=\tau_{\mu} e^{ \pm \frac{2.57 \tan \left[\frac{\alpha \pi}{2}\right]}{\alpha^{0.096}}}$ & $\tau_{ \pm}=\tau_{\mu} e^{ \pm \frac{5.52 \tan \left[\frac{\alpha \pi}{2}\right]}{\alpha^{0.30}}}$ \\
\hline log-normal & $\tau_{ \pm}=\tau_{\mu} e^{ \pm \frac{1.82 \sqrt{\alpha}}{1-\alpha}}$ & $\tau_{ \pm}=\tau_{\mu} e^{ \pm \frac{3.64 \sqrt{\alpha}}{1-\alpha}}$ \\
\hline
\end{tabular}

Conversely, Yager considered how a normal distribution of relaxation times on a logarithmic scale (lognormal distribution) could give rise to generalised Debye-like behaviour and tabulated values of the width parameter corresponding to certain values of $\alpha .{ }^{14}$ However, those expressions result in the outof-phase peak position moving as a function of the width of the distribution. Hence, we redefine the log-normal distribution such that the observed mean relaxation time $\tau_{\mu}$ is invariant to the width of the distribution (Equations 7 and 8). The $\gamma$ parameter controls the width of the distribution and there is the same population in the interval $\tau_{\mu} e^{-n \gamma} \leq \tau \leq \tau_{\mu} e^{n \gamma}$ as within $n \sigma$ for the normal distribution. By equating the peak value of $\chi^{\prime \prime}$ between the log-normal distribution model and the generalised Debye model (Equation 3 and 8), we can find the correspondence between $\gamma$ and $\alpha$ (Figure 4). We observe that $\gamma$ rises rapidly for small values of $\alpha$ and becomes approximately linear for $\alpha>0.06$, before rising quickly for $\alpha>0.7$ when the distribution becomes infinitely broad as $\alpha \rightarrow 1$. As before, there is no analytical solution to this problem, however the curve is very well approximated by Equation 9 (again, note that this is simply an interpolating function with the correct limiting behaviour 
and not derived from the distribution). Note that fitting the Fuoss-Kirkwood data with the form of Equation 9 is not as good as with Equation 6 (Figure S2 cf. Figures 3 and S1), and also that these lognormal data are not as well replicated with the form of Equation 6 (Figure $\mathrm{S} 3 \mathrm{cf}$. Figure 4).

$$
\begin{aligned}
\rho_{\text {log-normal }}(\tau, \gamma) & =\frac{1}{\tau \gamma \sqrt{2 \pi}} e^{-\frac{1}{2}\left(\frac{\ln [\tau]-\ln [\tau]}{\gamma}\right)^{2}} \\
\chi_{\text {Peak;log-normal }}^{\prime \prime}(\gamma) & =\int_{\tau \rightarrow 0}^{\tau=\infty} \rho_{\text {log-normal }}(\tau, \gamma)\left(\frac{\left(\chi_{T}-\chi_{S}\right) \frac{\tau}{\tau_{\mu}}}{1+\left(\frac{\tau}{\tau_{\mu}}\right)^{2}}\right) d \tau
\end{aligned}
$$

$$
\gamma \approx \frac{1.82 \sqrt{\alpha}}{1-\alpha}
$$
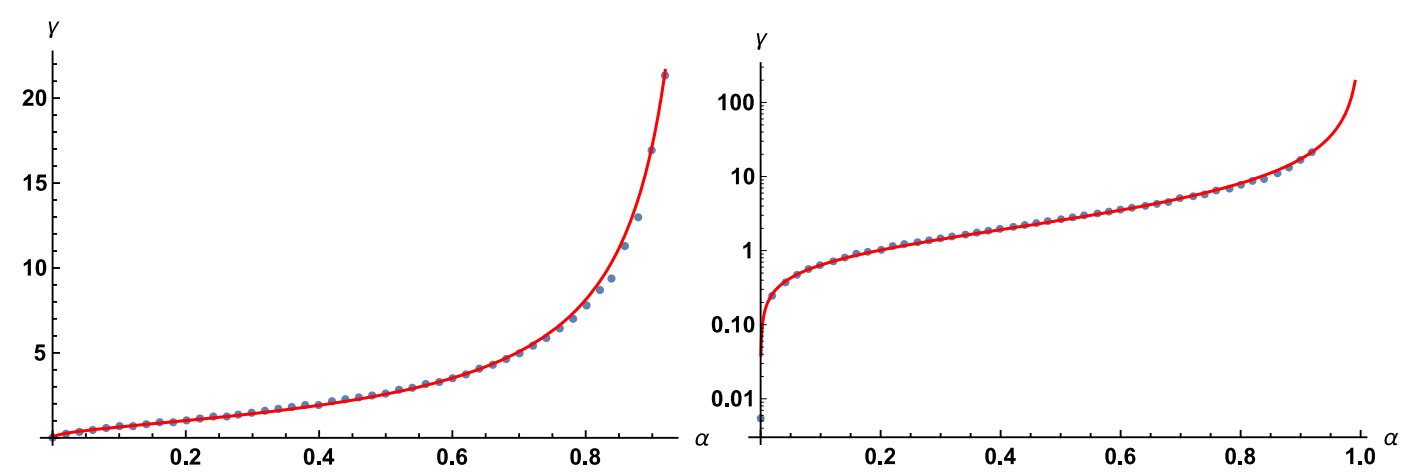

Figure 4 - Equivalence of $\gamma$ as a function of $\alpha$ on a linear $y$-scale (left) and a logarithmic $y$-scale (right). Red lines are a best fit with $\gamma \approx \frac{1.82 \sqrt{\alpha}}{1-\alpha}$.

With this equivalence in hand we can compare the AC magnetic susceptibility for the log-normal distribution to the generalised Debye model; recall that the latter implies the Fuoss-Kirkwood distribution (Figure 5). For small values of $\alpha$ the two models are practically indistinguishable, and as $\alpha$ increases we observe a slight compression at the extremities in the log-normal model. Comparing the parameter uncertainties, the two models suggest very similar ranges at the $1 \sigma$ level, while the FuossKirkwood model suggests larger ranges at the $2 \sigma$ level (Figure S4). Given that the log-normal model shows excellent agreement with the generalised Debye model (Figure 5) and that it can be simply extended to arbitrary $n \sigma$ levels, we suggest that it is the best model for considering uncertainties in AC magnetometry. 

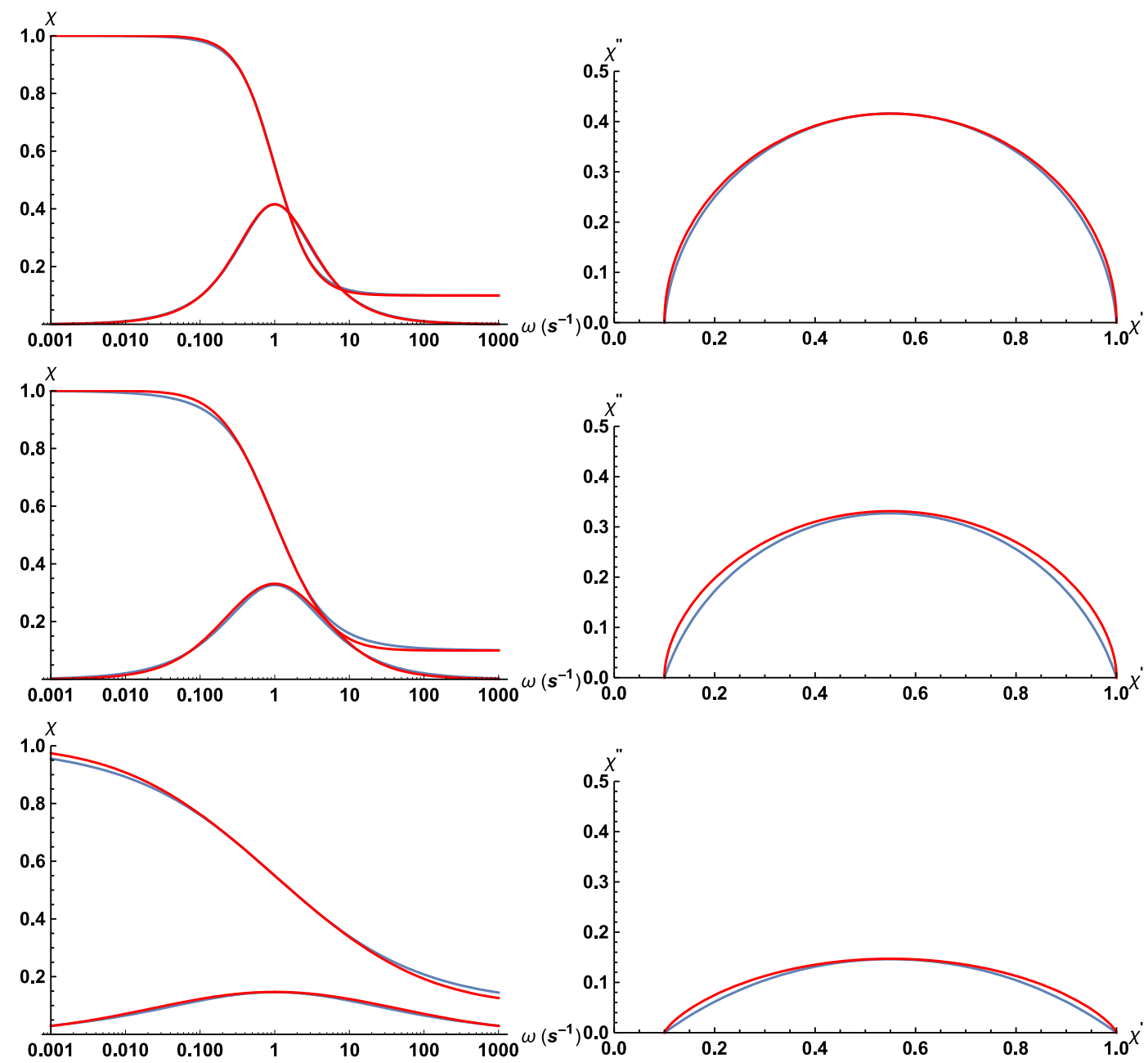

Figure 5 - Comparison of the AC magnetic susceptibility for the generalised Debye model (blue), and the log-normal distribution model (red). In-phase and out-of-phase AC magnetic susceptibility $\chi$ as a function of $\omega$ (left column), and out-of-phase AC magnetic susceptibility $\chi^{\prime \prime}$ as a function of the inphase AC magnetic susceptibility $\chi^{\prime}$ (right column), for $\alpha=0.05$ (top row), $\alpha=0.2$ (middle row) and $\alpha=0.6$ (bottom row). $\chi_{S}=0.1, \chi_{T}=1$ and $\tau_{\mu}=1 \mathrm{~s}$.

\section{Implementation}

We have written a new program called CC-FIT2 for modelling AC susceptibility data with the generalised Debye model, that determines uncertainties in the magnetic relaxation times as described herein, and furthermore provides provision for fitting the relaxation data with those uncertainties included. CC-FIT2 is available for free at http://www.nfchilton.com/cc-fit, and can be run on Windows, MacOS and Linux. The program takes as input a Quantum Design MultiVu ac.dat file and automatically selects the datasets that show clear peaks in the out-of-phase susceptibility $\chi^{\prime \prime}$ (i.e. with measurable relaxation times). The user is shown the data and prompted to select which model should be used to fit the data: the Debye, generalised Debye, or a combination of two generalised Debye models are available. When a model is chosen, the AC data are automatically fitted and the magnetic relaxation rates are displayed with $1 \sigma$ uncertainties derived from the log-normal distribution model (these data are also saved to disk). The user is then asked to choose a model for fitting the temperature dependence of the relaxation rates, from a combination of Orbach, Raman and QTM processes, and the relaxation rate data are fitted accounting for uncertainties with a variant of Equation 10 depending 
on the chosen model (note that $10^{A}=\tau_{0}, 10^{R}=C$ and $10^{Q}=\tau_{Q T M}$ ). In cases where $\alpha \rightarrow 0$, the main uncertainty in $\tau$ does not come from a distribution of $\tau$, but from the uncertainty in fitting the model function (i.e. the generalised Debye model) to the AC data; we call this $\tau_{\text {err }}$. To accommodate this situation, we define the uncertainty in the fitting procedure with Equation 11.

$$
\begin{gathered}
\log _{10}\left[\tau^{-1}\right]=\log _{10}\left[10^{-A} e^{-U_{e f f} / T}+10^{R} T^{n}+10^{-Q}\right] \\
\sigma_{\tau}=\max \left[\begin{array}{l}
\left|\log _{10}\left[\tau^{-1}\right]-\log _{10}\left[\tau_{\mu} e^{+\frac{1.82 \sqrt{\alpha}}{1-\alpha}}\right]\right|,\left|\log _{10}\left[\tau^{-1}\right]-\log _{10}\left[\tau_{\mu} e^{-\frac{1.82 \sqrt{\alpha}}{1-\alpha}}\right]\right|, \\
\left|\log _{10}\left[\tau^{-1}\right]-\log _{10}\left[\left(\tau+\tau_{\text {err }}\right)^{-1}\right]\right|,\left|\log _{10}\left[\tau^{-1}\right]-\log _{10}\left[\left(\tau-\tau_{e r r}\right)^{-1}\right]\right|
\end{array}\right]
\end{gathered}
$$

\section{Discussion}

We are now in a position to interpret experimental $\alpha$ values; here we examine a series of highperformance SMMs with large $U_{\text {eff }}$ barriers from the literature (Table 2; note that herein we employ the literature-reported $\alpha$ values and have not re-fit the underlying AC data). Firstly we discuss $\left[\mathrm{Dy}\left({ }^{\mathrm{t}} \mathrm{BuO}\right)_{2}(\mathrm{py})_{5}\right]\left[\mathrm{B}(\mathrm{Ph})_{4}\right]$ and $\left[\mathrm{Dy}\left({ }^{\mathrm{t}} \mathrm{BuO}\right) \mathrm{Cl}(\mathrm{THF})_{5}\right]\left[\mathrm{B}(\mathrm{Ph})_{4}\right]$ (py = pyridine, THF = tetrahydrofuran) $)^{5,7}$ These two molecules are structurally similar, both featuring a linear arrangement of formal charge along a single axis $\mathrm{O}^{-}-\mathrm{Dy}^{3+}-\mathrm{O}^{-} / \mathrm{Cl}^{-}$and five neutral equatorial ligands. The linear arrangement of charge induces a large magnetic anisotropy for the central Dy(III) ion, stabilising the largest $m_{J}= \pm \frac{15}{2}$ projections in the ground state and leading to a large $U_{\text {eff }}$ barrier in both cases. The barrier is substantially larger for $\left[\mathrm{Dy}\left({ }^{\mathrm{t}} \mathrm{BuO}\right)_{2}(\mathrm{py})_{5}\right]\left[\mathrm{B}(\mathrm{Ph})_{4}\right]$ than for $\left[\mathrm{Dy}\left({ }^{\mathrm{t}} \mathrm{BuO}\right) \mathrm{Cl}(\mathrm{THF})_{5}\right]\left[\mathrm{B}(\mathrm{Ph})_{4}\right]$ due to the presence of two short Dy-O bonds and a pair of stronger donor atoms $\left(\mathrm{O}^{-}>\mathrm{Cl}\right)$. None-the-less, the relaxation dynamics fitted using the generalised Debye model for the two samples give very different $\alpha$ values: for $\left[\mathrm{Dy}\left({ }^{\mathrm{t} B u O}\right)_{2}(\mathrm{py})_{5}\right]\left[\mathrm{B}(\mathrm{Ph})_{4}\right] \quad \alpha<0.02$ for all temperatures, while for $\left[\mathrm{Dy}\left({ }^{ } \mathrm{BuO}\right) \mathrm{Cl}(\mathrm{THF})_{5}\right]\left[\mathrm{B}(\mathrm{Ph})_{4}\right] \alpha$ varies over an order of magnitude in the range $0.03<\alpha<0.3$.

Using the log-normal model described above we convert the $\alpha$ values into uncertainty ranges for $\tau$ (any $\alpha$ values reported as $\sim 0$ are fixed at the arbitrarily small value of 0.001 so as not to over-bias the fit; CC-FIT2 does not apply any such criterion), and fit the data accounting for $1 \sigma$ uncertainties. Due to the small $\alpha$ values for $\left[\mathrm{Dy}\left({ }^{\mathrm{t}} \mathrm{BuO}\right)_{2}(\mathrm{py})_{5}\right]\left[\mathrm{B}(\mathrm{Ph})_{4}\right]$, there is not a large change in the level of uncertainty in the relaxation parameters with and without consideration of the distribution in $\tau$ (Figure 6, left; Table 2). However, due to the substantially larger $\alpha$ values for $\left[\mathrm{Dy}\left({ }^{\mathrm{t}} \mathrm{BuO}\right) \mathrm{Cl}(\mathrm{THF})_{5}\right]\left[\mathrm{B}(\mathrm{Ph})_{4}\right]$, the uncertainties in the relaxation parameters change significantly when a distribution in $\tau$ is accounted for (Figure 6, right; Table 2). For instance, $U_{\text {eff }}$ is only defined within $\pm 100 \mathrm{~K}, \tau_{0}$ covers two orders of magnitude, the Raman coefficient $C$ spans four orders of magnitude, and the Raman exponent $n$ varies from 2 to 6; clearly this has huge consequences for any comparison between this molecule and others, or to the underlying physics of the relaxation mechanisms.

It also raises the question as to why, for two such chemically similar molecules, is there such a significant difference in the distributions of their magnetic relaxation times. In an attempt to answer this question, we have examined the single crystal X-ray diffraction (XRD) structures collected at 100 $K$. We observe that the isotropic displacement parameters $\left(U_{\text {iso }}\right)$ for the atoms in the first coordination sphere are slightly larger for $\left[\mathrm{Dy}\left({ }^{\mathrm{t}} \mathrm{BuO}\right) \mathrm{Cl}(\mathrm{THF})_{5}\right]\left[\mathrm{B}(\mathrm{Ph})_{4}\right](\mathrm{Dy} 1=2.132(7), \mathrm{O} 1=3.60(9), \mathrm{Cl} 1=2.72(2), \mathrm{O} 2$ $=3.37(8), \mathrm{O} 3=3.48(8), \mathrm{O} 4=2.17(6), \mathrm{O} 5=2.78(7), \mathrm{O} 6=2.86(7) ;$ all $10^{-2} \AA^{2}$ ) than for 
$\left[\mathrm{Dy}\left({ }^{\mathrm{t} B u O}\right)_{2}(\mathrm{py})_{5}\right]\left[\mathrm{B}(\mathrm{Ph})_{4}\right](\mathrm{Dy} 1=1.968(6), \mathrm{O} 1=2.56(5), \mathrm{O} 2=2.51(5), \mathrm{N} 1=2.26(6), \mathrm{N} 2=2.36(6), \mathrm{N} 3=$ 2.64(6), N4 = 2.55(6), N5 = 2.29(6); all $\left.10^{-2} \AA^{2}\right)$; the anisotropic displacement parameters can be visualised as ellipsoids (Figure 6 bottom), clearly showing larger displacements for $\left[\mathrm{Dy}\left({ }^{\mathrm{t}} \mathrm{BuO}\right) \mathrm{Cl}(\mathrm{THF})_{5}\right]\left[\mathrm{B}(\mathrm{Ph})_{4}\right]$. We acknowledge that bulk properties cannot necessarily be inferred from single crystal data, but these results may indeed point to an origin of the broader distribution for the latter molecule.
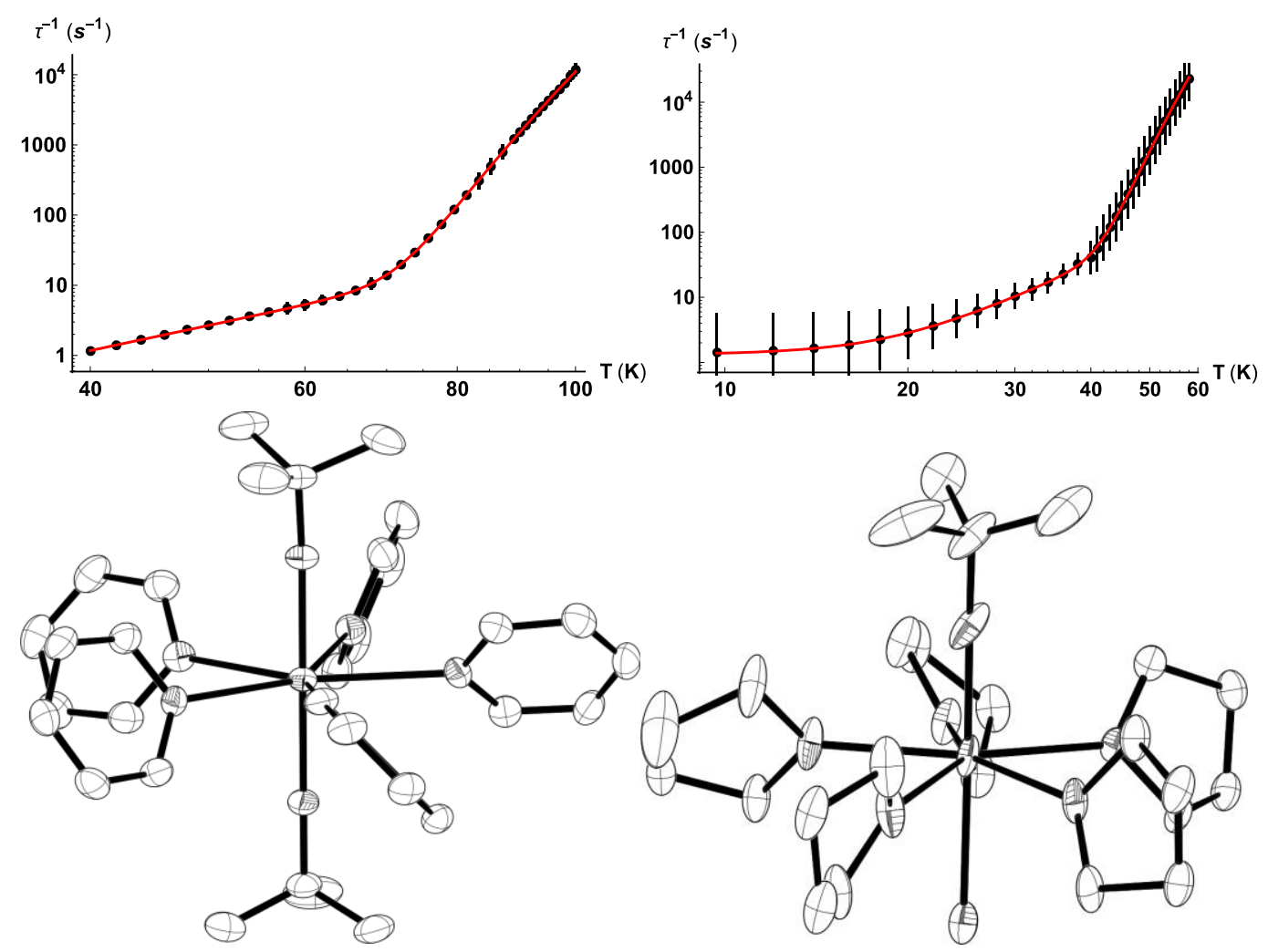

Figure 6 - Comparison of the relaxation dynamics for $\left[\mathrm{Dy}\left({ }^{\mathrm{t} B u O}\right)_{2}(\mathrm{py})_{5}\right]\left[\mathrm{B}(\mathrm{Ph})_{4}\right]$ (top left) and $\left[\mathrm{Dy}\left({ }^{\mathrm{t}} \mathrm{BuO}\right) \mathrm{Cl}(\mathrm{THF})_{5}\right]\left[\mathrm{B}(\mathrm{Ph})_{4}\right]$ (top right), and the crystal structures of the cationic complexes $\left[\mathrm{Dy}\left({ }^{\mathrm{t}} \mathrm{BuO}\right)_{2}(\mathrm{py})_{5}\right]^{+}$(bottom left) and $\left[\mathrm{Dy}\left({ }^{\mathrm{t}} \mathrm{BuO}\right) \mathrm{Cl}(\mathrm{THF})_{5}\right]^{+}$(bottom right). Error bars are calculated using the log-normal distribution model at the $1 \sigma$ level. Red lines are fits to Equation 10 with the $1 \sigma$ level parameters given in Table 2 . Ellipsoids shown with $50 \%$ probability at $100 \mathrm{~K}$, hydrogen atoms omitted for clarity.

Table 2 - Magnetic relaxation parameters accounting for distributions in the relaxation time. First row for each compound is without considering any uncertainty in the $\tau$ values (i.e. $0 \sigma$ ). Numbers in parentheses are standard errors (i.e. $1 \sigma$ parameter ranges).

\begin{tabular}{|c|c|c|c|c|c|c|}
\hline Compound & Range & $U_{e f f}(\mathrm{~K})$ & $A(\log [s])$ & $R\left(\log \left[\mathrm{s}^{-1} \mathrm{~K}^{-n}\right]\right)$ & $n$ & $Q(\log [s])$ \\
\hline \multirow{2}{*}{$\begin{array}{c}{\left[\mathrm{Dy}\left({ }^{\mathrm{t} B u O}\right)_{2}(\mathrm{py})_{5}\right]} \\
{\left[\mathrm{B}(\mathrm{Ph})_{4}\right]^{7}}\end{array}$} & $0 \sigma$ & 1812(4) & $-11.92(2)$ & $-5.86(5)$ & $3.70(3)$ & - \\
\hline & $1 \sigma$ & $1800(20)$ & $-11.88(9)$ & $-5.8(2)$ & $3.7(1)$ & - \\
\hline \multirow{2}{*}{$\begin{array}{c}{\left[\mathrm{Dy}\left({ }^{\mathrm{t}} \mathrm{BuO}\right) \mathrm{Cl}(\mathrm{THF})_{5}\right]} \\
{\left[\mathrm{B}(\mathrm{Ph})_{4}\right]^{5}}\end{array}$} & $0 \sigma$ & $957(6)$ & $-11.54(5)$ & $-5.4(1)$ & $4.32(8)$ & $-0.12(1)$ \\
\hline & $1 \sigma$ & $1000(100)$ & $-12(1)$ & $-6(2)$ & $4(2)$ & $-0.1(4)$ \\
\hline [Dy(bbpen)Cl] $^{6}$ a & $0 \sigma$ & $920(10)$ & $-11.8(1)$ & $-2.97(2)$ & $3.55(2)$ & $-0.358(6)$ \\
\hline
\end{tabular}




\begin{tabular}{|c|c|c|c|c|c|c|}
\hline & $1 \sigma$ & $900(200)$ & $-12(2)$ & $-3.0(6)$ & $3.5(4)$ & $-0.4(4)$ \\
\hline \multirow{2}{*}[\mathrm{Dy}(\text{bbpen})\mathrm{Br}]{$^{6 \mathrm{a}}$} & $0 \sigma$ & $1120(10)$ & $-12.04(9)$ & $-4.05(3)$ & $3.71(2)$ & - \\
\hline & $1 \sigma$ & $1110(80)$ & $-12.0(6)$ & $-4.1(3)$ & $3.7(2)$ & - \\
\hline \multirow{2}{*}{$\begin{array}{c}{\left[\mathrm{Dy}\left\{\mathrm{Cp}\left({ }^{\mathrm{t}} \mathrm{Bu}\right)_{3}\right\}_{2}\right]} \\
{\left[\mathrm{B}\left(\mathrm{C}_{6} \mathrm{~F}_{5}\right)_{4}\right]^{8}}\end{array}$} & $0 \sigma$ & $1760(20)$ & $-10.7(1)$ & - & - & - \\
\hline & $1 \sigma$ & $1780(40)$ & $-10.8(1)$ & - & - & - \\
\hline \multirow{2}{*}{$\begin{array}{c}{\left[\mathrm{Dy}\left\{\mathrm{Cp}(\mathrm{Me})_{5}\right\}\left\{\mathrm{Cp}\left({ }^{\prime} \mathrm{Pr}\right)_{5}\right\}\right]} \\
{\left[\mathrm{B}\left(\mathrm{C}_{6} \mathrm{~F}_{5}\right)_{4}\right]^{9}}\end{array}$} & $0 \sigma$ & $2250(10)$ & $-11.50(6)$ & - & - & - \\
\hline & $1 \sigma$ & $2230(10)$ & $-11.45(5)$ & - & - & - \\
\hline \multirow{2}{*}{$\begin{array}{c}{\left[\mathrm{Dy}\left\{\mathrm{Cp}\left({ }^{(} \mathrm{Pr}\right)_{4}\right\}_{2}\right]} \\
{\left[\mathrm{B}\left(\mathrm{C}_{6} \mathrm{~F}_{5}\right)_{4}\right]^{16}}\end{array}$} & $0 \sigma$ & $1870(20)$ & $-11.55(9)$ & - & - & - \\
\hline & $1 \sigma$ & $1850(20)$ & $-11.47(9)$ & - & - & - \\
\hline \multirow{2}{*}{$\begin{array}{c}{\left[\mathrm{Dy}\left\{\mathrm{Cp}\left(\mathrm{CPr}_{4}(\mathrm{Me})\right\}_{2}\right]\right.} \\
{\left[\mathrm{B}\left(\mathrm{C}_{6} \mathrm{~F}_{5}\right)_{4}\right]^{16}}\end{array}$} & $\overline{0 \sigma}$ & $2050(20)$ & $-11.1(1)$ & - & - & - \\
\hline & $1 \sigma$ & $2000(100)$ & $-11.0(6)$ & - & - & - \\
\hline \multirow{2}{*}{$\begin{array}{c}{\left[\mathrm{Dy}\left\{\mathrm{Cp}\left({ }^{\prime} \mathrm{Pr}\right)_{4}(\mathrm{Et})\right\}_{2}\right]} \\
{\left[\mathrm{B}\left(\mathrm{C}_{6} \mathrm{~F}_{5}\right)_{4}\right]^{16}}\end{array}$} & $0 \sigma$ & $1950(20)$ & $-10.9(1)$ & - & - & - \\
\hline & $1 \sigma$ & $2000(100)$ & $-11.0(7)$ & - & - & - \\
\hline \multirow{2}{*}{$\begin{array}{c}{\left[\mathrm{Dy}\left\{\mathrm{Cp}\left(\mathrm{iPr}_{5}\right\}_{2}\right]\right.} \\
{\left[\mathrm{B}\left(\mathrm{C}_{6} \mathrm{~F}_{5}\right)_{4}\right]^{16}}\end{array}$} & $0 \sigma$ & $1890(20)$ & $-10.81(9)$ & - & - & - \\
\hline & $1 \sigma$ & $1900(200)$ & $-10.8(7)$ & - & - & - \\
\hline
\end{tabular}

${ }^{a} \mathrm{H}_{2}$ bbpen = N,N'-bis(2-hydroxybenzyl)-N,N'-bis(2-methylpyridyl)ethylenediamine

Examining another pair of high-performance SMMs from the literature that also feature pentagonal bipyramidal Dy(III) centres with short axial bonds, [Dy(bbpen)Cl] and [Dy(bbpen) Br] $\left(\mathrm{H}_{2}\right.$ bbpen $=\mathrm{N}, \mathrm{N}^{\prime}-$ bis(2-hydroxybenzyl)-N,N'-bis(2-methylpyridyl)ethylenediamine), ${ }^{6}$ reveals that both show a steady increase in $\alpha$ values upon decreasing temperature (Figure 7); $\alpha<0.1$ for $T>20 \mathrm{~K}$, but increases rapidly below $20 \mathrm{~K}$ to reach $c a .0 .3$ at $5 \mathrm{~K}$ for [Dy(bbpen)Cl] (Figure S5). The trend of $\alpha$ with temperature is similar for both compounds, however the values for the $\mathrm{Cl}^{-}$analogue are around $3-5$ times larger than those of the $\mathrm{Br}^{-}$analogue between 20 and $50 \mathrm{~K}$ (Figure S5). This has a significant impact on the uncertainties in the fitted relaxation parameters: the uncertainties for [Dy(bbpen)Cl] are approximately double those for [Dy(bbpen)Br]. We note that this is not due to the larger distributions of $\tau$ in the low temperature (QTM) region for [Dy(bbpen)Cl], because near-identical parameters and uncertainties are obtained when fitting only the Orbach and Raman regions (Figure S6; Table S1). Examination of the XRD data for the two compounds (collected at $150 \mathrm{~K}$ ) again reveals that the $\mathrm{Cl}^{-}$ compound with larger $\alpha$ values also has larger $U_{\text {iso }}$ values for the atoms in the first coordination sphere (Dy1 = 1.543(9), $\mathrm{O} 1=2.11(9), \mathrm{Cl}=3.25(4), \mathrm{N} 1=1.80(11), \mathrm{N} 2=2.16(11)$; all $10^{-2} \AA^{2}$ ) than for the $\mathrm{Br}^{-}$ compound (Dy1 = 1.481(9), O1 = 1.96(7), Br1 = 2.811(15), N1 = 1.61(8), N2 = 2.14(8); all $10^{-2} \AA^{2}$ ).
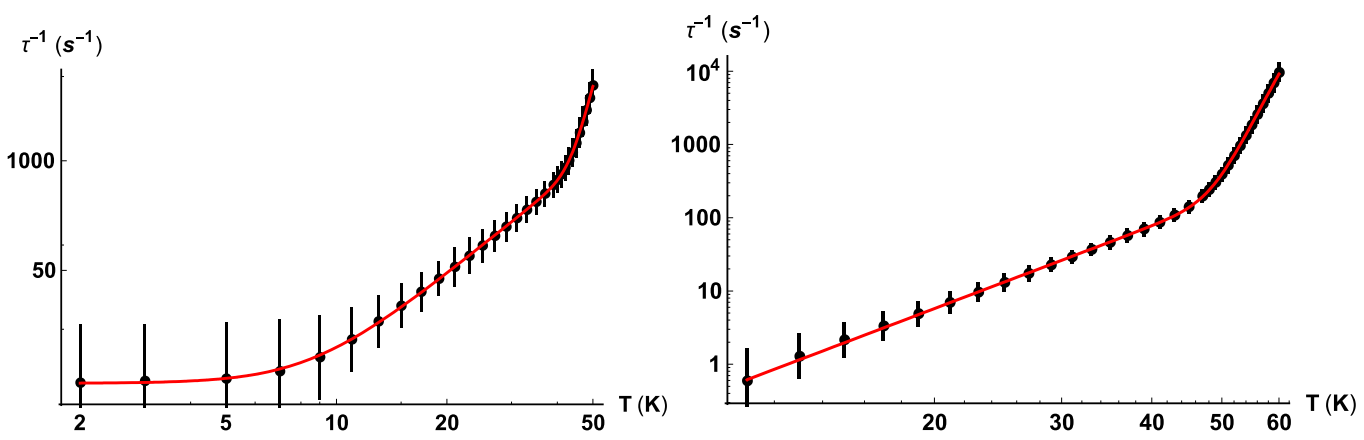

Figure 7 - Comparison of the relaxation dynamics for [Dy(bbpen)Cl] (left) and [Dy(bbpen)Br] (right), note the log-log scale. Error bars are calculated using the log-normal distribution model at the $1 \sigma$ level. Red lines are fits to Equation 10 with the $1 \sigma$ level parameters given in Table 2. 
Turning our attention to the recent family of dysprosocenium cations, ${ }^{8,9,16}$ the compounds appear to fall into two categories: the original $\left[\mathrm{Dy}\left\{\mathrm{Cp}\left({ }^{\mathrm{t}} \mathrm{Bu}_{3}\right\}_{2}\right]\left[\mathrm{B}\left(\mathrm{C}_{6} \mathrm{~F}_{5}\right)_{4}\right],{ }^{8}\right.$ the more recent tetra-substituted $\left[\operatorname{Dy}\left\{\mathrm{Cp}\left({ }^{(} \mathrm{Pr}\right)_{4}\right\}_{2}\right]\left[\mathrm{B}\left(\mathrm{C}_{6} \mathrm{~F}_{5}\right)_{4}\right],{ }^{16}$ and the fully substituted $\left[\mathrm{Dy}\left\{\mathrm{Cp}(\mathrm{Me})_{5}\right\}\left\{\mathrm{Cp}\left({ }^{\mathrm{P} r} \mathrm{Pr}\right)_{5}\right\}\right]\left[\mathrm{B}\left(\mathrm{C}_{6} \mathrm{~F}_{5}\right)_{4}\right],{ }^{9}$ have very small $\alpha$ values of $<0.05$ (originally fitted with fixed $\alpha=0$ in ref. ${ }^{8}$, we have re-fit the AC data here), $<0.04$ and $<0.03$, respectively, however the remaining members of the $\left[\mathrm{Dy}\left\{\mathrm{Cp}(\mathrm{Pr})_{4} \mathrm{R}\right\}_{2}\right]\left[\mathrm{B}\left(\mathrm{C}_{6} \mathrm{~F}_{5}\right)_{4}\right](\mathrm{R}=\mathrm{Me}, \mathrm{Et}$, iPr) series ${ }^{16}$ have significantly larger $\alpha$ values with maximal values around 0.1 to 0.15 . This means that the relaxation parameters are very well-defined for the former set of compounds (Figure 8 top row; Table 2), where $U_{\text {eff }}$ varies by $10^{\prime}$ s $K$ and $\tau_{0}$ varies within $1 / 10^{\text {th }}$ an order of magnitude, however the uncertainties for the latter set are significantly larger such that the relaxation parameters for the three compounds cannot be distinguished within error (Figure 8 bottom row and Figure S7; Table 2). Examination of the XRD structures for these six compounds (data collected at 100 or $150 \mathrm{~K}$ ) shows that there is substantial disorder and large displacement parameters for $\left[\mathrm{Dy}\left\{\mathrm{Cp}\left({ }^{(} \mathrm{Pr}\right)_{4} \mathrm{R}\right\}_{2}\right]\left[\mathrm{B}\left(\mathrm{C}_{6} \mathrm{~F}_{5}\right)_{4}\right](\mathrm{R}=$ $\mathrm{Me}, \mathrm{Et}$, $\mathrm{Pr}$; Figure 9 bottom row), while there is no disorder for $\left[\mathrm{Dy}\left\{\mathrm{Cp}\left({ }^{\mathrm{t}} \mathrm{Bu} \mathrm{u}_{3}\right\}_{2}\right]\left[\mathrm{B}\left(\mathrm{C}_{6} \mathrm{~F}_{5}\right)_{4}\right]\right.$ and $\left.\operatorname{Dy}\left\{\mathrm{Cp}\left({ }^{\mathrm{PPr}}\right)_{4}\right\}_{2}\right]\left[\mathrm{B}\left(\mathrm{C}_{6} \mathrm{~F}_{5}\right)_{4}\right]$, and minimal disorder for $\left[\mathrm{Dy}\left\{\mathrm{Cp}(\mathrm{Me})_{5}\right\}\left\{\mathrm{Cp}(\mathrm{PPr})_{5}\right\}\right]\left[\mathrm{B}\left(\mathrm{C}_{6} \mathrm{~F}_{5}\right)_{4}\right]$ (Figure 9 top row).
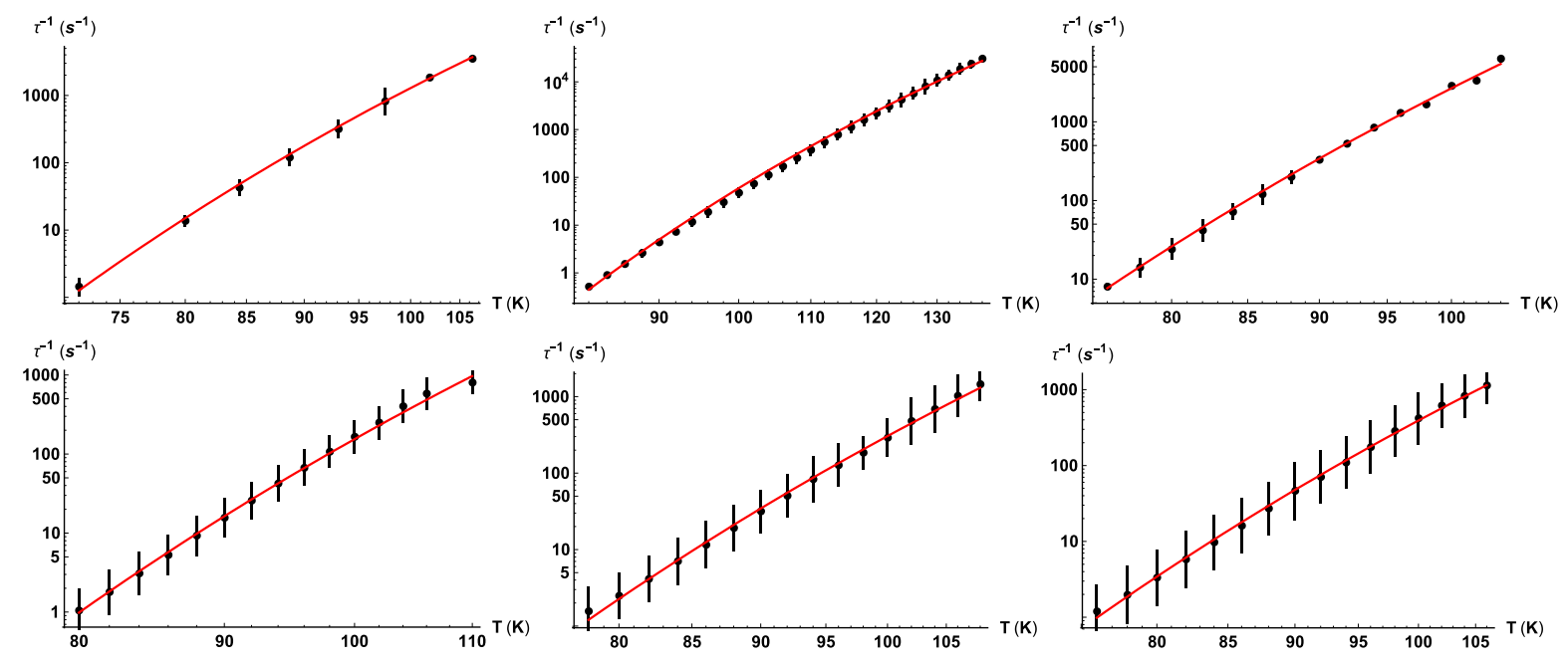

Figure 8 - Comparison of the relaxation dynamics for $\left[\mathrm{Dy}\left\{\mathrm{Cp}\left({ }^{\mathrm{t}} \mathrm{Bu}\right)_{3}\right\}_{2}\right]\left[\mathrm{B}\left(\mathrm{C}_{6} \mathrm{~F}_{5}\right)_{4}\right]$ (top left), $\left[\mathrm{Dy}\left\{\mathrm{Cp}(\mathrm{Me})_{5}\right\}\left\{\mathrm{Cp}\left({ }^{\mathrm{P} P r}\right)_{5}\right\}\right]\left[\mathrm{B}\left(\mathrm{C}_{6} \mathrm{~F}_{5}\right)_{4}\right] \quad$ (top middle), $\left[\mathrm{Dy}\left\{\mathrm{Cp}\left({ }^{(} \mathrm{Pr}\right)_{4}\right\}_{2}\right]\left[\mathrm{B}\left(\mathrm{C}_{6} \mathrm{~F}_{5}\right)_{4}\right]$ (top right), $\left[\mathrm{Dy}\left\{\mathrm{Cp}\left({ }^{(} \mathrm{Pr}\right)_{4} \mathrm{Me}\right\}_{2}\right]\left[\mathrm{B}\left(\mathrm{C}_{6} \mathrm{~F}_{5}\right)_{4}\right] \quad$ (bottom left), $\quad\left[\mathrm{Dy}\left\{\mathrm{Cp}\left({ }^{(} \mathrm{Pr}\right)_{4} \mathrm{Et}\right\}_{2}\right]\left[\mathrm{B}\left(\mathrm{C}_{6} \mathrm{~F}_{5}\right)_{4}\right] \quad$ (bottom middle) and $\left[\mathrm{Dy}\left\{\mathrm{Cp}\left({ }^{(} \mathrm{Pr}\right)_{5}\right\}_{2}\right]\left[\mathrm{B}\left(\mathrm{C}_{6} \mathrm{~F}_{5}\right)_{4}\right]$ (bottom right), note the log-log scale. Error bars are calculated using the lognormal distribution model at the $1 \sigma$ level. Red lines are fits to Equation 10 with the $1 \sigma$ level parameters given in Table 2. 

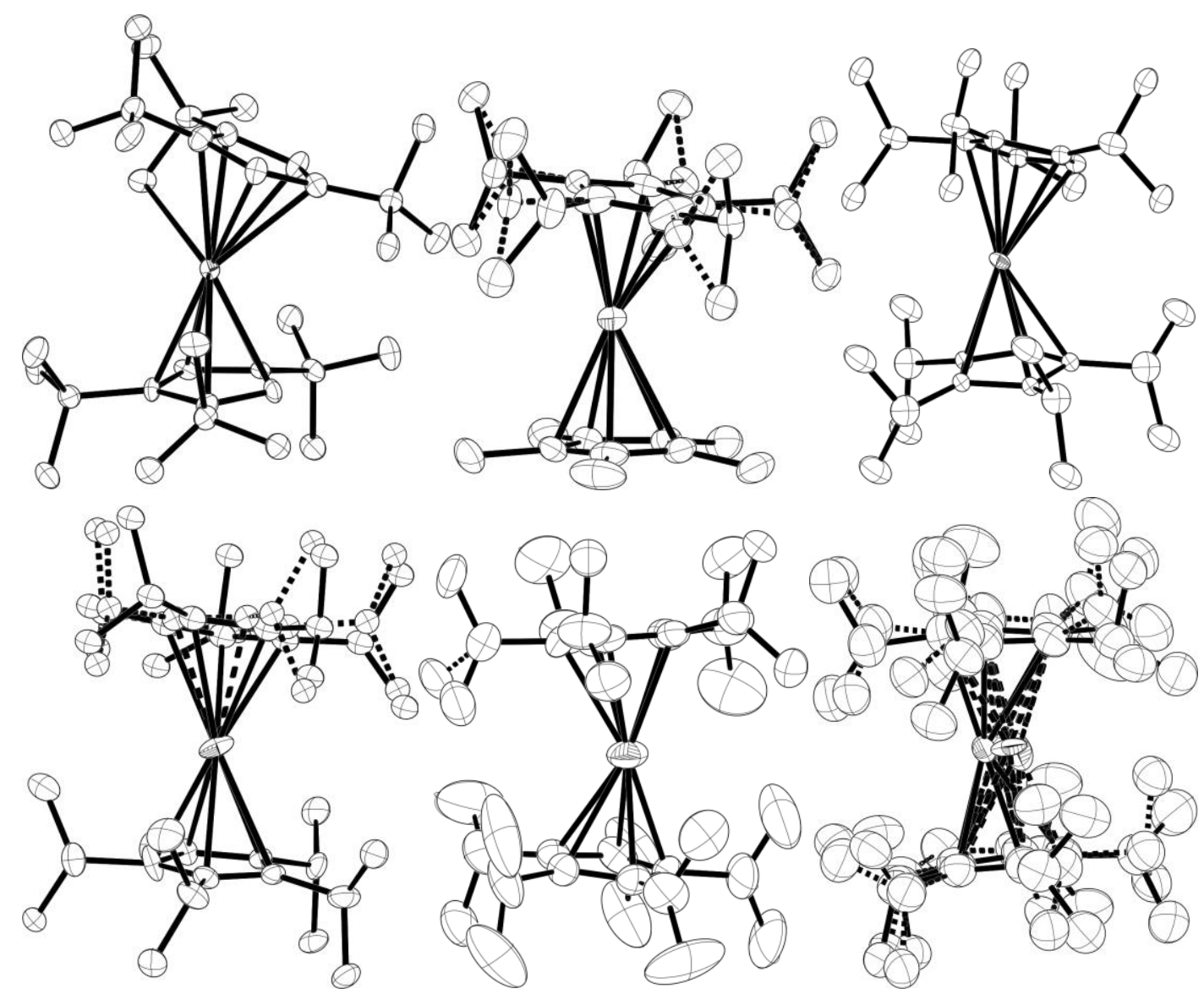

Figure 9 - Comparison of the crystal structures of the cationic complexes $\left[\mathrm{Dy}\left\{\mathrm{Cp}\left({ }^{\mathrm{t} B u}\right)_{3}\right\}_{2}\right]^{+}$(top left), $\left[\mathrm{Dy}\left\{\mathrm{Cp}(\mathrm{Me})_{5}\right\}\left\{\mathrm{Cp}\left(\text { ('Pr }_{5}\right\}\right]^{+}\right.$(top middle), $\left[\mathrm{Dy}\left\{\mathrm{Cp}\left({ }^{(\mathrm{Pr} r}\right)_{4}\right\}_{2}\right]^{+}$(top right), $\left[\mathrm{Dy}\left\{\mathrm{Cp}\left({ }^{(} \mathrm{Pr}\right)_{4} \mathrm{Me}\right\}_{2}\right]^{+}$(bottom left), $\left[\mathrm{Dy}\left\{\mathrm{Cp}\left({ }^{\mathrm{P} P r}\right)_{4} \mathrm{Et}\right\}_{2}\right]^{+}$(bottom middle) and $\left[\mathrm{Dy}\left\{\mathrm{Cp}\left({ }^{(} \mathrm{Pr}\right)_{5}\right\}_{2}\right]^{+}$(bottom right). Ellipsoids shown with $50 \%$ probability at $150 \mathrm{~K}$ for $\left.\mathrm{Dy}\left\{\mathrm{Cp}\left({ }^{\mathrm{t}} \mathrm{Bu}\right)_{3}\right\}_{2}\right]\left[\mathrm{B}\left(\mathrm{C}_{6} \mathrm{~F}_{5}\right)_{4}\right]$ and $\left[\mathrm{Dy}\left\{\mathrm{Cp}(\mathrm{Me})_{5}\right\}\left\{\mathrm{Cp}\left({ }^{(} \mathrm{Pr}\right)_{5}\right\}\right]\left[\mathrm{B}\left(\mathrm{C}_{6} \mathrm{~F}_{5}\right)_{4}\right]$, and $100 \mathrm{~K}$ for $\left[\mathrm{Dy}\left\{\mathrm{Cp}(\mathrm{P} P)_{4} \mathrm{R}\right\}_{2}\right]\left[\mathrm{B}\left(\mathrm{C}_{6} \mathrm{~F}_{5}\right)_{4}\right](\mathrm{R}=\mathrm{H}, \mathrm{Me}, \mathrm{Et}$, i $\mathrm{Pr})$. Structures with dotted lines show disorder conformers, hydrogen atoms omitted for clarity.

\section{Conclusions}

Herein we have derived expressions for the uncertainties in magnetic relaxation times in terms of $\alpha$ values when obtained from fitting AC susceptibility data with the generalised Debye model. We find that a log-normal distribution in $\tau$ gives AC data that are practically identical to the generalised Debye model, and that the $1 \sigma$ uncertainty ranges for this distribution are equivalent to the Fuoss-Kirkwood distribution which is directly derived from the generalised Debye model. Given these equivalences and the facile extension to arbitrary $n \sigma$ levels, we recommend use of the log-normal model. We have also presented a new freely available program CC-FIT2 for the fitting of AC susceptibility data, extraction of relaxation times with uncertainties, and modelling of relaxation data accounting for those uncertainties. Applying our approach to the magnetic relaxation data for a series of highperformance Dy(III) SMMs, we find that $\alpha$ values in the range of only 0.1 to 0.15 are large enough to result in uncertainties in $U_{\text {eff }}$ values of 100 's of $K$, and significantly larger relative uncertainties are obtained for the Raman parameters when $\alpha$ increases at lower temperatures. Interestingly, we observe markedly different $\alpha$ values even for families of similar complexes. Notably, we find that the relaxation parameters for $\left[\mathrm{Dy}\left\{\mathrm{Cp}\left({ }^{(} \mathrm{Pr}\right)_{4} \mathrm{R}\right\}_{2}\right]\left[\mathrm{B}\left(\mathrm{C}_{6} \mathrm{~F}_{5}\right)_{4}\right]\left(\mathrm{R}=\mathrm{Me}\right.$, Et, $\left.{ }^{\mathrm{P}} \mathrm{Pr}\right)$ cannot be distinguished within 
error, while those for $\left[\mathrm{Dy}\left\{\mathrm{Cp}\left({ }^{\mathrm{t}} \mathrm{Bu}\right\}_{3}\right\}_{2}\right]\left[\mathrm{B}\left(\mathrm{C}_{6} \mathrm{~F}_{5}\right)_{4}\right], \quad\left[\mathrm{Dy}\left\{\mathrm{Cp}\left({ }^{\mathrm{P} P r}\right)_{4}\right\}_{2}\right]\left[\mathrm{B}\left(\mathrm{C}_{6} \mathrm{~F}_{5}\right)_{4}\right]$, and $\left[\mathrm{Dy}\left\{\mathrm{Cp}(\mathrm{Me})_{5}\right\}\left\{\mathrm{Cp}\left({ }^{i} \mathrm{Pr}\right)_{5}\right\}\right]\left[\mathrm{B}\left(\mathrm{C}_{6} \mathrm{~F}_{5}\right)_{4}\right]$ are well-defined. Analysing the XRD data for the three families, we observe that larger $\alpha$ values tend to correlate with larger displacement parameters and/or crystallographic disorder, suggesting a physical origin for the distributions of relaxation times. We suggest that the type of analyses herein should become routine in the molecular magnetism community such that we can compare and contrast the behaviour of different complexes in a statistically meaningful way.

\section{Acknowledgements}

We thank The University of Manchester (Presidential Fellowship to NFC) and EPSRC (EP/R02605X/1) for funding, Prof. Ming-Liang Tong and Prof. Jeffrey Long for providing magnetic relaxation data, and Prof. Richard Winpenny and Prof. David Collison for useful conversations.

\section{References}

1 D. Gatteschi, R. Sessoli and J. Villain, Molecular Nanomagnets, Oxford University Press, 2006.

2 A. Abragam and B. Bleaney, Electron Paramagnetic Resonance of Transition Ions, Oxford University Press, 1970.

3 L. T. A. Ho and L. F. Chibotaru, Phys. Rev. B, 2018, 97, 024427.

4 L. Escalera-Moreno, J. J. Baldoví, A. Gaita-Ariño and E. Coronado, Chem. Sci., 2018, 9, 3265-3275.

5 Y.-S. Ding, K.-X. Yu, D. Reta, F. Ortu, R. E. P. Winpenny, Y.-Z. Zheng and N. F. Chilton, Nat. Commun., 2018, 9, 3134.

6 J. Liu, Y.-C. Chen, J.-L. Liu, V. Vieru, L. Ungur, J.-H. Jia, L. F. Chibotaru, Y. Lan, W. Wernsdorfer, S. Gao, X.-M. Chen and M.-L. Tong, J. Am. Chem. Soc., 2016, 138, 5441-5450.

7 Y.-S. Ding, N. F. Chilton, R. E. P. Winpenny and Y.-Z. Zheng, Angew Chem Int Ed, 2016, 55, 1607116074.

8 C. A. P. Goodwin, F. Ortu, D. Reta, N. F. Chilton and D. P. Mills, Nature, 2017, 548, 439-442.

9 F.-S. Guo, B. M. Day, Y.-C. Chen, M.-L. Tong, A. Mansikkamäki and R. A. Layfield, Science, 2018, 362, 1400-1403.

10 M. J. Giansiracusa, A. K. Kostopoulos, D. Collison, R. E. P. Winpenny and N. F. Chilton, Chem. Commun., , DOI:10.1039/C9CC02421B.

11 J.-L. Liu, Y.-C. Chen and M.-L. Tong, Chem. Soc. Rev., 2018, 47, 2431-2453.

12 K. S. Cole and R. H. Cole, J. Chem. Phys., 1941, 9, 341-351.

13 R. M. Fuoss and J. G. Kirkwood, J. Am. Chem. Soc., 1941, 63, 385-394.

14 W. A. Yager, Physics, 1936, 7, 434-450.

15 S. K. Gupta, T. Rajeshkumar, G. Rajaraman and R. Murugavel, Chem Sci, 2016, 7, 5181-5191.

16 K. R. McClain, C. A. Gould, K. Chakarawet, S. Teat, T. J. Groshens, J. R. Long and B. G. Harvey,

Chem. Sci., 2018, 9, 8492-8503. 